\title{
FURTHER RESULTS ON DIFFERENTIAL INEQUALITY OF A CLASS OF SECOND ORDER NEUTRAL TYPE
}

\author{
PEIGUANG WANG AND YONGHONG WU
}

\begin{abstract}
In this paper, we develop several new results related to the nonexistence criteria for eventually positive solutions of a class of second order neutral differential inequalities with distributed deviating arguments. The work generalizes various existing result.
\end{abstract}

\section{Introduction}

We consider the following second order neutral equations with distributed deviating arguments

$$
\begin{aligned}
& {[x(t)+c(t) x(t-\tau)]^{\prime \prime}+\int_{a}^{b} p(t, \xi) f(x[g(t, \xi)]) d \sigma(\xi) \leq 0,} \\
& {[x(t)+c(t) x(t-\tau)]^{\prime \prime}+\int_{a}^{b} p(t, \xi) f(x[g(t, \xi)]) d \sigma(\xi) \geq 0}
\end{aligned}
$$

where $\tau>0$ is a constant; $c(t) \in C\left(\left[t_{0}, \infty\right), I\right), I=[0,1] ; f(x) \in C(R, R)$ and $x f(x)>0$, for $x \neq 0, p(t, \xi) \in C\left(\left[t_{0}, \infty\right) \times[a, b], R_{+}\right)$, and $p(t, \xi)$ is not eventually zero on any ray $\left[t_{\mu}, \infty\right) \times[a, b], t_{\mu} \geq t_{0}, R_{+}=[0, \infty) ; g(t, \xi) \in C\left(\left[t_{0}, \infty\right) \times[a, b], R\right), \frac{d}{d t} g(t, a)$ exists, $g(t, \xi) \leq t, \xi \in[a, b] ; g(t, \xi)$ is nondecreasing with respect to $t$ and $\xi$ respectively; and $\liminf _{t \rightarrow \infty, \xi \in[a, b]}\{g(t, \xi)\}=\infty ; \sigma(\xi) \in([a, b], R)$ is nondecreasing, the integral of equation (1) is a Stieltjes one.

Recently, there has been an increasing interest in delay differential inequalities, and a number of results have been obtained. For more details, we refer the reader to the literature [1-5]. In this paper, we establish some general nonexistence criteria of eventually positive solutions for inequality $(1)$.

As is customary, the solution $x(t) \in C\left(\left[t_{0}, \infty\right), R\right)$ of inequality (1) is said to be eventually positive if there exists a sufficiently large positive number $\mu$ such that the inequality $x(t)>0$ holds for $t \geq \mu$.

Received May 7, 2002.

2000 Mathematics Subject Classification. 34K10, 34K15.

Key words and phrases. differential inequality, neutral equation, distributed deviating arguments, eventually positive solution. 


\section{Nonexistence Criteria}

The following theorems provide the sufficient conditions leading to nonexistence of eventually positive solutions for inequality (1).

Theorem 1. Assume that $f(-x)=-f(x), x \in(0, \infty)$, and

$$
\frac{f(x)}{x} \geq \lambda, \quad x \in(0, \infty), \quad \text { for some constant } \lambda>0 .
$$

If for any integer $m>2$,

$$
\limsup _{t \rightarrow \infty} \frac{1}{t^{m}} \int_{t_{0}}^{t}\left[\lambda(t-s)^{m} \int_{a}^{b} p(s, \xi)\{1-c[g(s, \xi)]\} d \sigma(\xi)-\frac{m^{2}(t-s)^{2}}{4 g^{\prime}(s, a)}\right] d s=\infty,
$$

then inequality (1) has no eventually positive solutions.

Proof. Assume the contrary, without loss of generality, $x(t)$ is an eventually positive solution of inequality (1). Then from $\liminf _{t \rightarrow \infty, \xi \in[a, b]}\{g(t, \xi)\}=\infty$, there exists a $t_{1} \geq t_{0}$ such that $x(t)>0, x(t-\tau)>0$ and $x[g(t, \xi)]>0, t \geq t_{1}, \xi \in[a, b]$. Set

$$
y(t)=x(t)+c(t) x(t-\tau),
$$

then, we have $y(t) \geq x(t)>0, y^{\prime \prime}(t) \leq 0, t \geq t_{1}$, and we can claim that $y^{\prime}(t) \geq 0, t \geq t_{1}$. In fact, assume that it is not true, then there exists a $t_{2} \geq t_{1}$ such that $y^{\prime}\left(t_{2}\right)<0$. From the fact that $y^{\prime}(t)$ is decreasing, there exists a $t_{3} \geq t_{2}$ such that $y^{\prime}\left(t_{3}\right)<0$, and $y^{\prime}(t) \leq y^{\prime}\left(t_{3}\right)<0, t \geq t_{3}$. Integrating from $t_{3}$ to $t$, we have $y(t) \leq y\left(t_{3}\right)+y^{\prime}\left(t_{3}\right)\left(t-t_{3}\right)$. Thus, we conclude that $\lim _{t \rightarrow \infty} y(t)=-\infty$. This contradicts $y(t)>0$. From (1) and the condition of Theorem 1, we obtain

$$
\begin{aligned}
0 & \geq y^{\prime \prime}(t)+\int_{a}^{b} p(t, \xi) f(x[g(t, \xi)]) d \sigma(\xi) \\
& \geq y^{\prime \prime}(t)+\lambda \int_{a}^{b} p(t, \xi)\{y[g(t, \xi)]-c[g(t, \xi)] x[g(t, \xi)-\tau]\} d \sigma(\xi) .
\end{aligned}
$$

Using $y^{\prime}(t) \geq 0$, and $y(t) \geq x(t), t \geq t_{1}$, we have $y[g(t, \xi)] \geq y[g(t, \xi)-\tau] \geq x[g(t, \xi)-\tau]$, thus

$$
y^{\prime \prime}(t)+\lambda \int_{a}^{b} p(t, \xi)\{1-c[g(t, \xi)]\} y[g(t, \xi)] d \sigma(\xi) \leq 0, \quad t \geq t_{1} .
$$

Furthermore, as $g(t, \xi)$ is nondecreasing with respect to $\xi$, we have

$$
y^{\prime \prime}(t)+\lambda y[g(t, a)] \int_{a}^{b} p(t, \xi)\{1-c[g(t, \xi)]\} d \sigma(\xi) \leq 0, \quad t \geq t_{1} .
$$

Set

$$
z(t)=\frac{y^{\prime}(t)}{y[g(t, a)]} .
$$


Then $z(t) \geq 0$. From the fact that there exists a $\frac{d}{d t} g(t, a)$, we obtain $y^{\prime}[g(t, a)]=$ $\frac{d y}{d g} \frac{d}{d t} g(t, a)$. Further, by noting that $g(t, \xi)$ is nondecreasing with respect to $\xi, g(t, \xi) \leq t$, $\xi \in[a, b]$, and $y^{\prime \prime}(t) \leq 0$, we obtain $y^{\prime}(t) \leq y^{\prime}[g(t, a)]$. Thus

$$
\begin{aligned}
z^{\prime}(t) & =\frac{y^{\prime \prime}(t)}{y[g(t, a)]}-\frac{y^{\prime}(t) y^{\prime}[g(t, a)] g^{\prime}(t, a)}{y^{2}[g(t, a)]} \\
& \leq-\lambda \int_{a}^{b} p(t, \xi)\{1-c[g(t, \xi)]\} d \sigma(\xi)-g^{\prime}(t, a) z^{2}(t), \quad t \geq t_{1} .
\end{aligned}
$$

Integrating by parts for any $t>T \geq t_{1}$, we have

$$
\begin{aligned}
& \int_{T}^{t} \lambda(t-s)^{m} \int_{a}^{b} p(s, \xi)\{1-c[g(s, \xi)]\} d \sigma(\xi) d s \\
\leq & -\int_{T}^{t}(t-s)^{m} z^{\prime}(s) d s-\int_{T}^{t}(t-s)^{m} g^{\prime}(s, a) z^{2}(s) d s \\
= & -\int_{T}^{t}(t-s)^{m} d z(s)-\int_{T}^{t}(t-s)^{m} g^{\prime}(s, a) z^{2}(s) d s \\
= & (t-T)^{m} z(T)-m \int_{T}^{t}(t-s)^{m-1} z(s) d s-\int_{T}^{t}(t-s)^{m} g^{\prime}(s, a) z^{2}(s) d s \\
= & (t-T)^{m} z(T)-\int_{T}^{t}\left[\sqrt{g^{\prime}(s, a)(t-s)^{m}} z(s)+\frac{m(t-s)^{\frac{m}{2}-1}}{2 \sqrt{g^{\prime}(s, a)}}\right]^{2} d s+\int_{T}^{t} \frac{m^{2}(t-s)^{m-2}}{4 g^{\prime}(s, a)} d s,
\end{aligned}
$$

which implies that for $t>T \geq t_{0}$

$$
\begin{aligned}
& \int_{T}^{t}\left[\lambda(t-s)^{m} \int_{a}^{b} p(s, \xi)\{1-c[g(s, \xi)]\} d \sigma(\xi)-\frac{m^{2}(t-s)^{m-2}}{4 g^{\prime}(s, a)}\right] d s \\
\leq & (t-T)^{m} z(T)-\int_{T}^{t}\left[\sqrt{g^{\prime}(s, a)(t-s)^{m}} z(s)+\frac{m(t-s)^{\frac{m}{2}-1}}{2 \sqrt{g^{\prime}(s, a)}}\right]^{2} d s .
\end{aligned}
$$

Furthermore, we have

$$
\begin{aligned}
& \int_{t_{1}}^{t}\left[\lambda(t-s)^{m} \int_{a}^{b} p(s, \xi)\{1-c[g(s, \xi)]\} d \sigma(\xi)-\frac{m^{2}(t-s)^{m-2}}{4 g^{\prime}(s, a)}\right] d s \\
\leq & \left(t-t_{1}\right)^{m} z\left(t_{1}\right)-\int_{t_{1}}^{t}\left[\sqrt{g^{\prime}(s, a)(t-s)^{m}} z(s)+\frac{m(t-s)^{\frac{m}{2}-1}}{2 \sqrt{g^{\prime}(s, a)}}\right]^{2} d s \\
\leq & \left(t-t_{1}\right)^{m} z\left(t_{1}\right) \leq\left(t-t_{0}\right)^{m} z\left(t_{1}\right) .
\end{aligned}
$$

Thus, we have that

$$
\frac{1}{t^{m}} \int_{t_{0}}^{t}\left[\lambda(t-s)^{m} \int_{a}^{b} p(s, \xi)\{1-c[g(s, \xi)]\} d \sigma(\xi)-\frac{m^{2}(t-s)^{m-2}}{4 g^{\prime}(s, a)}\right] d s
$$




$$
\begin{aligned}
& =\frac{1}{t^{m}}\left[\int_{t_{0}}^{t_{1}}+\int_{t_{1}}^{t}\right]\left[\lambda(t-s)^{m} \int_{a}^{b} p(s, \xi)\{1-c[g(s, \xi)]\} d \sigma(\xi)-\frac{m^{2}(t-s)^{m-2}}{4 g^{\prime}(s, a)}\right] d s \\
& \leq\left(1-\frac{t_{0}}{t}\right)^{m} z\left(t_{1}\right)+\int_{t_{0}}^{t_{1}} \lambda\left(1-\frac{s}{t}\right)^{m} \int_{a}^{b} p(s, \xi)\{1-c[g(s, \xi)]\} d \sigma(\xi) d s \\
& \leq z\left(t_{1}\right)+\lambda \int_{t_{0}}^{t_{1}} \int_{a}^{b} p(s, \xi)\{1-c[g(s, \xi)]\} d \sigma(\xi) d s
\end{aligned}
$$

which implies that

$$
\begin{aligned}
& \limsup _{t \rightarrow \infty} \frac{1}{t^{m}} \int_{t_{0}}^{t}\left[\lambda(t-s)^{m} \int_{a}^{b} p(s, \xi)\{1-c[g(s, \xi)]\} d \sigma(\xi)-\frac{m^{2}(t-s)^{m-2}}{4 g^{\prime}(s, a)}\right] d s \\
& \leq z\left(t_{1}\right)+\lambda \int_{t_{0}}^{t_{1}} \int_{a}^{b} p(s, \xi)\{1-c[g(s, \xi)]\} d \sigma(\xi) d s<\infty,
\end{aligned}
$$

this contradicts (4). Therefore, the proof of Theorem 1 is completed.

From the proof of Theorem 1, we have the following corollary.

Corollary 1. If the condition (4) of Theorem 1 is replaced by

$$
\begin{gathered}
\limsup _{t \rightarrow \infty} \frac{1}{t^{m}} \int_{t_{0}}^{t}(t-s)^{m} \int_{a}^{b} p(s, \xi)\{1-c[g(s, \xi)]\} d \sigma(\xi) d s=\infty, \\
\limsup _{t \rightarrow \infty} \frac{1}{t^{m}} \int_{t_{0}}^{t} \frac{(t-s)^{m-2}}{g^{\prime}(s, a)} d s<\infty
\end{gathered}
$$

then inequality (1) has no eventually positive solutions.

Theorem 2. Assume that the condition of (3) holds, and there exists a constant $m \geq 2$ and function $\rho(t) \in C^{\prime}\left(\left[t_{0}, \infty\right),(0, \infty)\right)$ such that

$$
\begin{gathered}
\limsup _{t \rightarrow \infty} \frac{1}{t^{m}} \int_{t_{0}}^{t} \frac{(t-s)^{m-2}}{\rho(s) g^{\prime}(s, a)}\left[m \rho(s)-(t-s) \rho^{\prime}(s)\right]^{2} d s<\infty \\
\limsup _{t \rightarrow \infty} \frac{1}{t^{m}} \int_{t_{0}}^{t}(t-s)^{m} \rho(s) \int_{a}^{b} p(s, \xi)\{1-c[g(s, \xi)]\} d \sigma(\xi) d s=\infty,
\end{gathered}
$$

then inequality (1) has no eventually positive solutions.

Proof. Assume the contrary, without loss of generality, that $x(t)$ is an eventually positive solution of inequality (1). Then proceeding as Theorem 1 , there exists a $t_{1} \geq t_{0}$ such that

$$
z^{\prime}(t) \leq-\lambda \int_{a}^{b} p(t, \xi)\{1-c[g(t, \xi)]\} d \sigma(\xi)-g^{\prime}(t, a) z^{2}(t), \quad t \geq t_{1}
$$


Thus

$$
\begin{aligned}
& \int_{t_{1}}^{t} \lambda(t-s)^{m} \rho(s) \int_{a}^{b} p(s, \xi)\{1-c[g(s, \xi)]\} d \sigma(\xi) d s \\
\leq & -\int_{t_{1}}^{t}(t-s)^{m} \rho(s) z^{\prime}(s) d s-\int_{t_{1}}^{t}(t-s)^{m} \rho(s) g^{\prime}(s, a) z^{2}(s) d s \\
= & \left(t-t_{1}\right)^{m} \rho\left(t_{1}\right) z\left(t_{1}\right)-\int_{t_{1}}^{t}(t-s)^{m-1}\left[m \rho(s)-(t-s) \rho^{\prime}(s)\right] z(s) d s \\
& -\int_{t_{1}}^{t}(t-s)^{m} \rho(s) g^{\prime}(s, a) z^{2}(s) d s .
\end{aligned}
$$

Furthermore, we conclude that

$$
\begin{aligned}
& \int_{t_{1}}^{t} \lambda(t-s)^{m} \rho(s) \int_{a}^{b} p(s, \xi)\{1-c[g(s, \xi)]\} d \sigma(\xi) d s \\
\leq & \left(t-t_{1}\right)^{m} \rho\left(t_{1}\right) z\left(t_{1}\right) \\
& -\int_{t_{1}}^{t}\left\{\sqrt{\rho(s) g^{\prime}(s, a)}(t-s)^{\frac{m}{2}} z(s)+\frac{(t-s)^{\frac{m}{2}-1}}{2 \sqrt{\rho(s) g^{\prime}(s, a)}}\left[m \rho(s)-(t-s) \rho^{\prime}(s)\right]\right\}^{2} d s \\
& +\frac{1}{4} \int_{t_{1}}^{t} \frac{(t-s)^{m-2}}{\rho(s) g^{\prime}(s, a)}\left[m \rho(s)-(t-s) \rho^{\prime}(s)\right]^{2} d s \\
\leq & \left(t-t_{1}\right)^{m} \rho\left(t_{1}\right) z\left(t_{1}\right)+\frac{1}{4} \int_{t_{1}}^{t} \frac{(t-s)^{m-2}}{\rho(s) g^{\prime}(s, a)}\left[m \rho(s)-(t-s) \rho^{\prime}(s)\right]^{2} d s .
\end{aligned}
$$

From (19), for $t>T \geq t_{1}$, we obtain

$$
\begin{aligned}
& \frac{1}{t^{m}} \int_{t_{1}}^{t} \lambda(t-s)^{m} \rho(s) \int_{a}^{b} p(s, \xi)\{1-c[g(s, \xi)]\} d \sigma(\xi) d s \\
= & \frac{1}{t^{m}}\left[\int_{t_{1}}^{T}+\int_{T}^{t}\right]\left[\lambda(t-s)^{m} \rho(s) \int_{a}^{b} p(s, \xi)\{1-c[g(s, \xi)]\}\right] d \sigma(\xi) d s \\
\leq & \frac{1}{t^{m}} \int_{t_{1}}^{T} \lambda(t-s)^{m} \rho(s) \int_{a}^{b} p(s, \xi)\{1-c[g(s, \xi)]\} d \sigma(\xi) d s+\frac{1}{t^{m}}\left(t-t_{1}\right)^{m} \rho\left(t_{1}\right) z\left(t_{1}\right) \\
& +\frac{1}{4 t^{m}} \int_{t_{1}}^{t} \frac{(t-s)^{m-2}}{\rho(s) g^{\prime}(s, a)}\left[m \rho(s)-(t-s) \rho^{\prime}(s)\right]^{2} d s
\end{aligned}
$$

which implies that

$$
\begin{aligned}
& \limsup _{t \rightarrow \infty} \frac{1}{t^{m}} \int_{t_{1}}^{t} \lambda(t-s)^{m} \rho(s) \int_{a}^{b} p(s, \xi)\{1-c[g(s, \xi)]\} d \sigma(\xi) d s \\
\leq & L+\frac{1}{4} \limsup _{t \rightarrow \infty} \frac{1}{t^{m}} \int_{t_{1}}^{t} \frac{(t-s)^{m-2}}{\rho(s) g^{\prime}(s, a)}\left[m \rho(s)-(t-s) \rho^{\prime}(s)\right]^{2} d s,
\end{aligned}
$$


where $L=\rho\left(t_{1}\right) z\left(t_{1}\right)$. Thus, from condition (16), we conclude that

$$
\limsup _{t \rightarrow \infty} \frac{1}{t^{m}} \int_{t_{1}}^{t}(t-s)^{m} \rho(s) \int_{a}^{b} p(s, \xi)\{1-c[g(s, \xi)]\} d \sigma(\xi) d s<\infty,
$$

which contradicts (17). Therefore, the proof of Theorem 2 is completed.

Theorem 3. Assume that the condition of (3) holds, and there exists a constant $m \geq 2$ and function $\rho(t) \in C^{\prime}\left(\left[t_{0}, \infty\right),(0, \infty)\right)$ such that

$$
\limsup _{t \rightarrow \infty} \frac{1}{t^{m}} \int_{t_{0}}^{t}(t-s)^{m} \rho(s) \int_{a}^{b} p(s, \xi)\{1-c[g(s, \xi)]\} d \sigma(\xi) d s<\infty,
$$

and there exists a function $\varphi(t) \in C\left(\left[t_{0}, \infty\right), R\right)$ satisfying

$$
\begin{aligned}
& \liminf _{t \rightarrow \infty} \frac{1}{t^{m}} \int_{u}^{t}\left[\lambda(t-s)^{m} \rho(s) \int_{a}^{b} p(s, \xi)\{1-c[g(s, \xi)]\} d \sigma(\xi)\right. \\
&\left.\quad-\frac{(t-s)^{m-2}}{4 \rho(s) g^{\prime}(s, a)}\left[m \rho(s)-(t-s) \rho^{\prime}(s)\right]^{2}\right] d s \geq \varphi(u), \quad u \geq t_{0}, \\
& \lim _{t \rightarrow \infty} \frac{1}{t^{m}} \int_{t_{0}}^{t} \frac{(t-s)^{m} g^{\prime}(s, a) \varphi_{+}^{2}(s)}{\rho(s)} d s=\infty, \quad \varphi_{+}(s)=\max _{s \geq t_{0}}\{\varphi(s), 0\}
\end{aligned}
$$

then inequality (1) has no eventually positive solutions.

Proof. Assume the contrary, without loss of generality, that $x(t)$ is an eventually positive solution of inequality (1). Then proceeding as for Theorem 2, there exists a $t_{1}>u \geq t_{0}$ such that

$$
\begin{aligned}
& \int_{u}^{t} \lambda(t-s)^{m} \rho(s) \int_{a}^{b} p(s, \xi)\{1-c[g(s, \xi)]\} d \sigma(\xi) d s \\
\leq & (t-u)^{m} \rho(u) z(u)+\frac{1}{4} \int_{u}^{t} \frac{(t-s)^{m-2}}{\rho(s) g^{\prime}(s, a)}\left[m \rho(s)-(t-s) \rho^{\prime}(s)\right]^{2} d s .
\end{aligned}
$$

Furthermore, for $t>u \geq t_{0}$, we have

$$
\begin{aligned}
\frac{1}{t^{m}} \int_{u}^{t}[ & \lambda(t-s)^{m} \rho(s) \int_{a}^{b} p(s, \xi)\{1-c[g(s, \xi)]\} d \sigma(\xi) \\
& \left.\quad-\frac{(t-s)^{m-2}}{4 \rho(s) g^{\prime}(s, a)}\left[m \rho(s)-(t-s) \rho^{\prime}(s)\right]^{2}\right] d s \leq \frac{1}{t^{m}}(t-u)^{m} \rho(u) z(u) .
\end{aligned}
$$

From (24), we conclude that

$$
\varphi(u) \leq \frac{1}{t^{m}} \int_{u}^{t}\left[\lambda(t-s)^{m} \rho(s) \int_{a}^{b} p(s, \xi)\{1-c[g(s, \xi)]\} d \sigma(\xi)\right.
$$




$$
\begin{aligned}
& \left.-\frac{(t-s)^{m-2}}{4 \rho(s) g^{\prime}(s, a)}\left[m \rho(s)-(t-s) \rho^{\prime}(s)\right]^{2}\right] d s \\
& \leq \rho(u) z(u),
\end{aligned}
$$

which implies that

$$
\varphi_{+}^{2}(u) \leq \rho^{2}(u) z^{2}(u)
$$

Let

$$
\begin{aligned}
& v(t)=\frac{1}{t^{m}} \int_{t_{1}}^{t} z(s)(t-s)^{m-1}\left[m \rho(s)-(t-s) \rho^{\prime}(s)\right] d s \\
& w(t)=\frac{1}{t^{m}} \int_{t_{1}}^{t} \rho(s) g^{\prime}(s, a)(t-s)^{m} z^{2}(s) d s
\end{aligned}
$$

then, from (20), we have

$$
v(t)+w(t) \leq \frac{1}{t^{m}}\left(t-t_{1}\right)^{m} \rho\left(t_{1}\right) z\left(t_{1}\right)-\lambda \int_{t_{1}}^{t}(t-s)^{m} \rho(s) \int_{a}^{b} p(s, \xi)\{1-c[g(s, \xi)]\} d \sigma(\xi) d s .
$$

Further, from (24), we have

$$
\begin{aligned}
& \liminf _{t \rightarrow \infty} \frac{1}{t^{m}} \int_{u}^{t} \lambda(t-s)^{m} \rho(s) \int_{a}^{b} p(s, \xi)\{1-c[g(s, \xi)]\} d \sigma(\xi) d s \geq \varphi(u), \\
& \limsup _{t \rightarrow \infty} \frac{1}{t^{m}} \int_{t_{1}}^{t} \lambda(t-s)^{m} \rho(s) \int_{a}^{b} p(s, \xi)\{1-c[g(s, \xi)]\} d \sigma(\xi) d s \\
& -\liminf _{t \rightarrow \infty} \frac{1}{t^{m}} \int_{t_{1}}^{t} \frac{(t-s)^{m-2}}{4 \rho(s) g^{\prime}(s, a)}\left[m \rho(s)-(t-s) \rho^{\prime}(s)\right]^{2} d s \geq \varphi\left(t_{1}\right) .
\end{aligned}
$$

From (30) and (23), we conclude that

$$
\liminf _{t \rightarrow \infty} \frac{1}{t^{m}} \int_{t_{1}}^{t} \frac{(t-s)^{m-2}}{4 \rho(s) g^{\prime}(s, a)}\left[m \rho(s)-(t-s) \rho^{\prime}(s)\right]^{2} d s<\infty .
$$

Thus, there exists a sequence $\left\{t_{n}\right\}_{1}^{\infty}$ in $\left(t_{1}, \infty\right)$ such that $\lim _{n \rightarrow \infty} t_{n}=\infty$ and

$$
\lim _{n \rightarrow \infty} \frac{1}{t_{n}^{m}} \int_{t_{1}}^{t_{n}} \frac{\left(t_{n}-s\right)^{m-2}}{4 \rho(s) g^{\prime}(s, a)}\left[m \rho(s)-\left(t_{n}-s\right) \rho^{\prime}(s)\right]^{2} d s<\infty,
$$

which implies that

$$
\begin{aligned}
& \limsup _{t \rightarrow \infty}\{v(t)+w(t)\} \\
\leq & \rho\left(t_{1}\right) z\left(t_{1}\right)-\liminf _{t \rightarrow \infty} \frac{1}{t^{m}} \int_{t_{1}}^{t} \lambda(t-s)^{m} \rho(s) \int_{a}^{b} p(s, \xi)\{1-c[g(s, \xi)]\} d \sigma(\xi) d s \\
\leq & \rho\left(t_{1}\right) z\left(t_{1}\right)-\varphi\left(t_{1}\right) \triangleq M .
\end{aligned}
$$


Hence, for any sufficiently large $n$, we have

$$
u\left(t_{n}\right)+v\left(t_{n}\right)<M_{1}
$$

where $M_{1}>M, M$ and $M_{1}$ are constant. From the definition of $w(t)$, we have

$$
w^{\prime}(t)=\int_{t_{1}}^{t} \frac{m s \rho(s) g^{\prime}(s, a)}{t^{2}}\left(1-\frac{s}{t}\right)^{m-1} z^{2}(s) d s>0 .
$$

Therefore, $w(t)$ is an increasing function, and $\lim _{t \rightarrow \infty} w(t)=l$ exists, where is either finite or infinite. In the case $l=\infty, \lim _{n \rightarrow \infty} w\left(t_{n}\right)=\infty$, which implies, from (33), that

$$
\lim _{n \rightarrow \infty} v\left(t_{n}\right)=-\infty
$$

and

$$
\frac{v\left(t_{n}\right)}{w\left(t_{n}\right)}+1>\frac{M_{1}}{w\left(t_{n}\right)}
$$

Thus, for any $0<\varepsilon<1$, and for any sufficiently large $n$, we have

$$
\frac{v\left(t_{n}\right)}{w\left(t_{n}\right)}<\varepsilon-1<0 .
$$

On the other hand, by using the Schwartz inequality, for $t \geq t_{1}$, we obtain

$$
\begin{aligned}
0 \leq & v^{2}\left(t_{n}\right)=\frac{1}{t_{n}^{2 m}}\left\{\int_{t_{1}}^{t_{n}} z(s)\left(t_{n}-s\right)^{m-1}\left[m \rho(s)-\left(t_{n}-s\right) \rho^{\prime}(s)\right] d s\right\}^{2} \\
\leq & \left\{\frac{1}{t_{n}^{m}} \int_{t_{1}}^{t_{n}} \rho(s) g^{\prime}(s, a)\left(t_{n}-s\right)^{m} z^{2}(s) d s\right\} \\
& \times\left\{\frac{1}{t_{n}^{m}} \int_{t_{1}}^{t_{n}} \frac{\left(t_{n}-s\right)^{m-2}}{\rho(s) g^{\prime}(s, a)}\left[m \rho(s)-\left(t_{n}-s\right) \rho^{\prime}(s)\right]^{2} d s\right\} \\
= & w\left(t_{n}\right) \frac{1}{t_{n}^{m}} \int_{t_{1}}^{t_{n}} \frac{\left(t_{n}-s\right)^{m-2}}{\rho(s) g^{\prime}(s, a)}\left[m \rho(s)-\left(t_{n}-s\right) \rho^{\prime}(s)\right]^{2} d s .
\end{aligned}
$$

Then

$$
0 \leq \frac{v^{2}\left(t_{n}\right)}{w\left(t_{n}\right)} \leq \frac{1}{t_{n}^{m}} \int_{t_{1}}^{t_{n}} \frac{\left(t_{n}-s\right)^{m-2}}{\rho(s) g^{\prime}(s, a)}\left[m \rho(s)-\left(t_{n}-s\right) \rho^{\prime}(s)\right]^{2} d s .
$$

It follows from (31) that

$$
0 \leq \lim _{n \rightarrow \infty} \frac{v^{2}\left(t_{n}\right)}{w\left(t_{n}\right)}<\infty
$$

From (35), we have

$$
\lim _{n \rightarrow \infty} \frac{v\left(t_{n}\right)}{w\left(t_{n}\right)}=\lim _{n \rightarrow \infty} \frac{v^{\prime}\left(t_{n}\right)}{w^{\prime}\left(t_{n}\right)} \leq \varepsilon-1<0,
$$

then

$$
\lim _{n \rightarrow \infty} \frac{v^{2}\left(t_{n}\right)}{w\left(t_{n}\right)}=\lim _{n \rightarrow \infty} \frac{2 v\left(t_{n}\right) v^{\prime}\left(t_{n}\right)}{w^{\prime}\left(t_{n}\right)} \geq 2 \lim _{n \rightarrow \infty} v\left(t_{n}\right)(\varepsilon-1)=\infty,
$$


which contradicts (37). Thus, we have $\lim _{t \rightarrow \infty} w(t)=c<\infty$. Furthermore, according to (28), we conclude that

$$
\begin{aligned}
\lim _{t \rightarrow \infty} \frac{1}{t^{m}} \int_{t_{1}}^{t} \frac{(t-s)^{m} g^{\prime}(s, a) \varphi_{+}^{2}(s)}{\rho(s)} d s & \leq \lim _{t \rightarrow \infty} \frac{1}{t^{m}} \int_{t_{1}}^{t} \rho(s) g^{\prime}(s, a)(t-s)^{m} z^{2}(s) d s \\
& =\lim _{t \rightarrow \infty} w(t)<\infty
\end{aligned}
$$

which implies that

$$
\begin{aligned}
\lim _{t \rightarrow \infty} \frac{1}{t^{m}} \int_{t_{0}}^{t} \frac{(t-s)^{m} g^{\prime}(s, a) \varphi_{+}^{2}(s)}{\rho(s)} d s & =\lim _{t \rightarrow \infty} \frac{1}{t^{m}}\left[\int_{t_{0}}^{t_{1}}+\int_{t_{1}}^{t}\right] \frac{(t-s)^{m} g^{\prime}(s, a) \varphi_{+}^{2}(s)}{\rho(s)} d s \\
& \leq \int_{t_{0}}^{t_{1}} \frac{(t-s)^{m} g^{\prime}(s, a) \varphi_{+}^{2}(s)}{\rho(s)} d s+\lim _{t \rightarrow \infty} w(t)<\infty
\end{aligned}
$$

which contradicts (25). Therefore, the proof of Theorem 3 is completed.

Similar to the above results on inequality (1), we can also obtain some results on inequality (2).

Theorem 4. Suppose that the conditions of Theorem 1 hold, then inequality (2) has no eventually negative solutions.

Theorem 5. Suppose that the conditions of Theorem 2 hold, then inequality (2) has no eventually negative solutions.

Theorem 6. Suppose that the conditions of Theorem 3 hold, then inequality (2) has no eventually negative solutions.

\section{References}

[1] G. Ladas and I. P. Stavroulakis, On delay differential inequalitites of first order, Funkcial. Ekvac. 25(1982), 105-113.

[2] J. Ruan, A class of differential inequalities with continuous distributed deviating arguments, Acta Math. Sinaca. 30(1987), 661-670. (in Chinese).

[3] X. Z. Liu and X. L. Fu, Nonlinear differential inequalities with distributed deviating arguments and applications, Nonlin. World. 1(1994), 409-427.

[4] L. Q. Zhang and X. L. Fu, A class of second order functional differential inequalities, Ann. Diff. Eqs. 12(1996), 129-133.

[5] P. G. Wang and W. G. Ge, Certain of second order second order differential inequality of neutral type, Appl. Math. Letters. 13(2000), 45-51.

College of Electronic and Information Engineering, Hebei University, Baoding, 071002, P.R. China.

E-mail: pgwang@mail.hbu.edu.cn

Department of Mathematics and Statistics, Curtin University of Technology, GPO Box U1987, Perth, Western Australia 6845, Australia. 